\title{
Ordered Nanoporous Silica Modified with Nanoparticles of Lanthanide Oxides
}

\author{
A. ZeleñÁKOVÁ ${ }^{a, *}$, O. KAPUsta ${ }^{b}, \mathrm{~V}$. ZeleñÁK ${ }^{b}$ \\ ${ }^{a}$ Institute of Physics, Faculty of Sciences, P.J. Šafárik University, Park Angelinum 9, 04154 Košice, Slovakia \\ ${ }^{b}$ Institute of Chemistry, Faculty of Sciences, P.J. Šafárik University, Moyzesova 11, 04154 Košice, Slovakia \\ Lanthanide oxide nanoparticles were encapsulated inside of pores of highly ordered periodic silica of SBA15 \\ type with hexagonal symmetry. The magnetic properties of such nanoperticles were investigated. The structural \\ characterization using the SAXS, XANES, XRD, and $\mathrm{N}_{2}$ adsorption measurements showed the presence of lan- \\ thanide oxides of $\mathrm{Ln}_{2} \mathrm{O}_{3}$ type ( $\mathrm{Ln}=\mathrm{La}, \mathrm{Pr}, \mathrm{Nd}, \mathrm{Gd}, \mathrm{Eu}$ ), with the size of about $5 \mathrm{~nm}$, incorporated in nanoporous \\ channel system. Their magnetic properties, studied by SQUID apparatus, showed the weak antiferromagnetic \\ ordering at $2 \mathrm{~K}$ in the nanocomposites $\mathrm{Gd}_{2} \mathrm{O}_{3} @$ SBA15, $\mathrm{Pr}_{2} \mathrm{O}_{3} @$ SBA15 and $\mathrm{Nd}_{2} \mathrm{O}_{3} @$ SBA15. This behaviour of the \\ nanoparticles is caused by blocking process of magnetic moments, which at $300 \mathrm{~K}$ exhibit the superparamagnetism, \\ evidenced from ZFC/FC magnetization.
}

DOI: 10.12693 /APhysPolA.126.218

PACS: 75.75.Fk, 81.05.Rm, 75.40.Cx

\section{Introduction}

Lanthanide metals have attracted much attention as suitable magnetic materials for a wide range of technological and biomedical applications [1]. For example, Gd and its compounds are of current interest as magnetic resonance contrast media, therapeutic agents in tumour treatment and drug delivery [2]. The well-defined shape and size of nanoparticles is necessary for applications. One of the promising methods of packing the magnetic nanoparticles into the ordered structures with the possibility of controlling their size and shape is the nanocasting approach [3].

In our work, we have studied the magnetic properties of $\mathrm{Ln}_{2} \mathrm{O}_{3}$ nanoparticles ( $\mathrm{Ln}=\mathrm{La}, \mathrm{Pr}, \mathrm{Nd}, \mathrm{Gd}, \mathrm{Eu}$ ) prepared inside a periodic porous silica matrix SBA-15, which served as a nanosized "mold" with regular hexagonal symmetry.

\section{Experimental}

Mesoporous material SBA-15 was synthesized according to Zhao, et al. [4]. Nanoparticles were prepared using wet-impregnation method, where the mesoporous silica template was used for the growth of the nanoparticles. The structure of prepared samples was characterized by SAXS (Small angle X-ray scattering), XANES (X-ray Near Edge Spectroscopy), powder X-ray diffraction and by using the method of $\mathrm{N}_{2}$ adsorption/desorption.

Magnetic properties were investigated using a MPMSXL5 (Quantum Design) apparatus in the external dc field of up to $5 \mathrm{~T}$ and in the temperature range of $2-300 \mathrm{~K}$.

\section{Results and discussion}

$\mathrm{N}_{2}$ sorption measurements, Fig. 1, show the decrease of the specific surface area $S_{B E T}$ from around $720 \mathrm{~m}^{2} / \mathrm{g}$,

*corresponding author; e-mail: adriana.zelenakova@upjs.sk observed for a blank mesoporous SBA15 matrix, to about $32-46 \mathrm{~m}^{2} / \mathrm{g}$ for $\mathrm{Ln}_{2} \mathrm{O}_{3} @$ SBA15 nanocomposites, due to the confinement of lanthanide oxide nanoparticles into mesoporous system (Table). This significant decrease of surface area reflects the filling of pores of SBA15 matrix with nanoparticles. Moreover, the filling of pores with nanoparticles was further reflected by the decrease of pore size $D_{p}$ from around $5.9 \mathrm{~nm}$ for blank SBA15 matrix to $5.2-5.4 \mathrm{~nm}$ for composite $\mathrm{Ln}_{2} \mathrm{O}_{3} @ \mathrm{SBA} 15 \mathrm{sam}-$ ples.

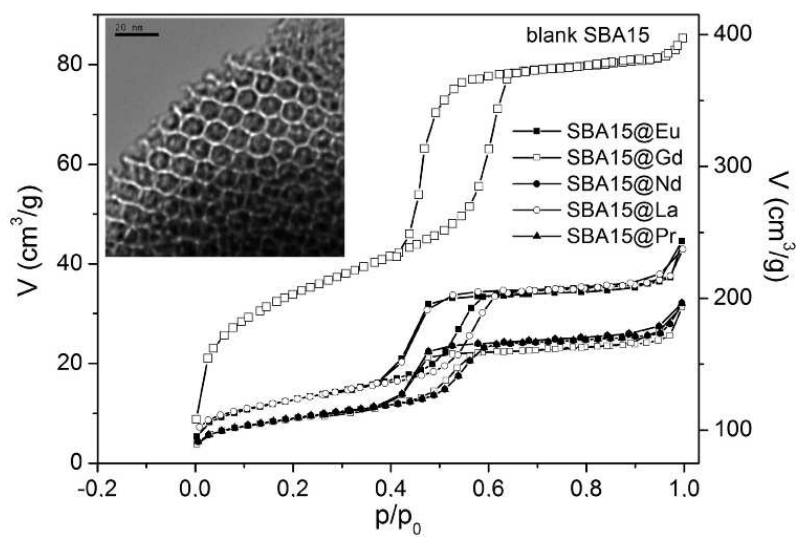

Fig. 1. Nitrogen adsorption/desorption isotherms of blank mesoporous silica (SBA15) and composite samples, containing lanthanide oxide nanoparticles at relative pressure. In the inset is the HRTEM picture of silica matrix.

The method of small angle X-ray scattering (SAXS) confirmed the hexagonal $p 6 \mathrm{~mm}$ symmetry of the blank mesoporous SBA15 silica matrix as well as of the composites. This showed that during the preparation of $\mathrm{Ln}_{2} \mathrm{O}_{3} @$ SBA15 nanocomposites no destruction or disordering of mesoporous hexagonal structure took place. 
Structural and magnetic parameters.

TABLE

\begin{tabular}{c|c|c|c|c|c}
\hline \hline Sample & $\begin{array}{c}S_{B E T} \\
\left(\mathrm{~m}^{2} / \mathrm{g}\right)\end{array}$ & $\begin{array}{c}D_{p} \\
(\mathrm{~nm})\end{array}$ & $\begin{array}{c}\theta \\
(\mathrm{K})\end{array}$ & $\begin{array}{c}\mu \\
\left(\mu_{B}\right)\end{array}$ & $\begin{array}{c}M_{S} \\
\left(\mathrm{~A} \cdot \mathrm{m}^{2} \mathrm{~kg}^{-1}\right)\end{array}$ \\
\hline $\mathrm{SBA15}$ & 719.4 & 5.9 & - & - & - \\
\hline $\mathrm{Gd}_{2} \mathrm{O}_{3} @ \mathrm{SBA} 15$ & 32.3 & 5.3 & -2.24 & 6.20 & 60.1 \\
\hline $\mathrm{Eu}_{2} \mathrm{O}_{3} @$ SBA15 & 46.0 & 5.3 & -252.93 & 1.74 & 1.1 \\
\hline $\mathrm{Pr}_{2} \mathrm{O}_{3} @$ SBA15 & 33.0 & 5.5 & -24.01 & 1.92 & 5.8 \\
\hline $\mathrm{Nd}_{2} \mathrm{O}_{3} @$ SBA15 & 33.0 & 5.5 & -33.96 & 4.83 & 16.5 \\
\hline $\mathrm{La}_{2} \mathrm{O}_{3} @$ SBA15 & 45.2 & 5.7 & - & - & -
\end{tabular}

Structural characterisation was further made by XANES and XRD measurements. XANES spectra show the presence of lanthanide oxides of $\mathrm{Ln}_{2} \mathrm{O}_{3}$ type in all modified samples. In Fig. 2 the representative spectra of Gd-based nanocomposite are presented. The XANES peak, measured at $\mathrm{Gd} L_{3}$ absorption edge, is narrower in the prepared sample than in the reference foil. This effect was observed in all lanthanide samples and could be explained by the nanocrystalline character of composite samples (about $5 \mathrm{~nm}$ ) in opposite to $\mu \mathrm{m}$ size of reference foil [5].

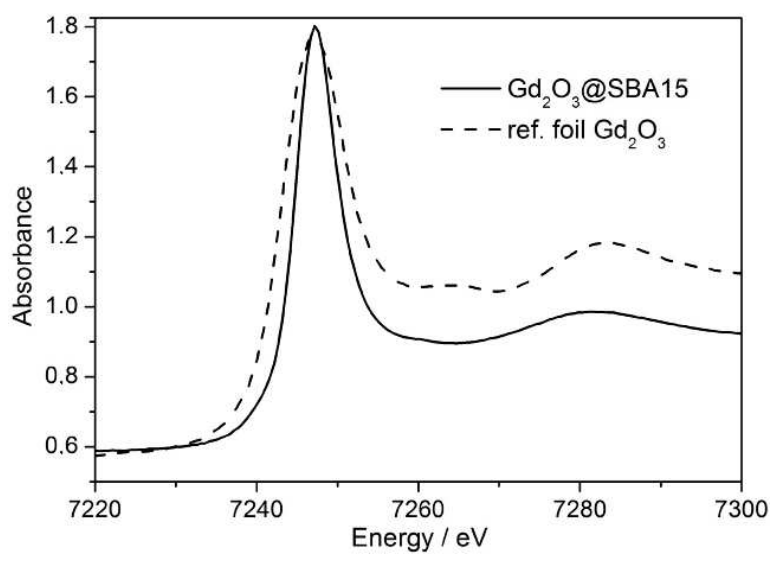

Fig. 2. XANES spectra for nanocomposite sample $\mathrm{Gd}_{2} \mathrm{O}_{3} @$ SBA15 and reference foil.

The magnetic properties of all lanthanide nanocomposite samples were investigated using the measurements of the temperature- and the field-dependences of magnetization. As it is seen from Fig. 3, the samples with $\mathrm{Gd}, \mathrm{Nd}, \mathrm{Pr}$ and Eu exhibit the reversible linear magnetization curve at $300 \mathrm{~K}$, predicting the paramagnetic behaviour, while the La-based nanocomposite is purely diamagnetic, as expected. The shape of the magnetization curves at $2 \mathrm{~K}$, especially in $\mathrm{Gd}$ nanocomposite, implies the presence of magnetic interactions at low temperatures. The dc susceptibility studies and their analysis using the Curie-Weiss law show the presence of weak antiferromagnetic interactions (see value of Weiss constant $\theta$ in Table). In the samples with $\mathrm{Gd}_{2} \mathrm{O}_{3}$ and $\mathrm{Nd}_{2} \mathrm{O}_{3}$ nanoparticles inside the porous silica matrix, the clearly evident superparamagnetic relaxation at high temperatures and blocking process of magnetic moments at low temperatures was observed from ZFC/FC curves and cur- vature of $M$ vs. $H$. Therefore we have analysed experimental data using Langevin law and calculated values of magnetic moments, which are given in the Table. The most interesting magnetic properties among all studied $\mathrm{Ln}_{2} \mathrm{O}_{3}$ nanocomposites exhibited the $\mathrm{Gd}_{2} \mathrm{O}_{3}$-containing sample, because of the high value of magnetic moment, high saturation magnetization $M_{S}$ and the presence of superparamagnetism (existence of blocking temperature $T_{B}$ around of $2.5 \mathrm{~K}$ ). In the sample with $\mathrm{Gd}_{2} \mathrm{O}_{3}$ the measured value of magnetic moment $\mu$ of $6.20 \mu_{B}$ is lower than the calculated theoretical value of $7.94 \mu_{B}$, which is due to nanosized character of the sample [2].

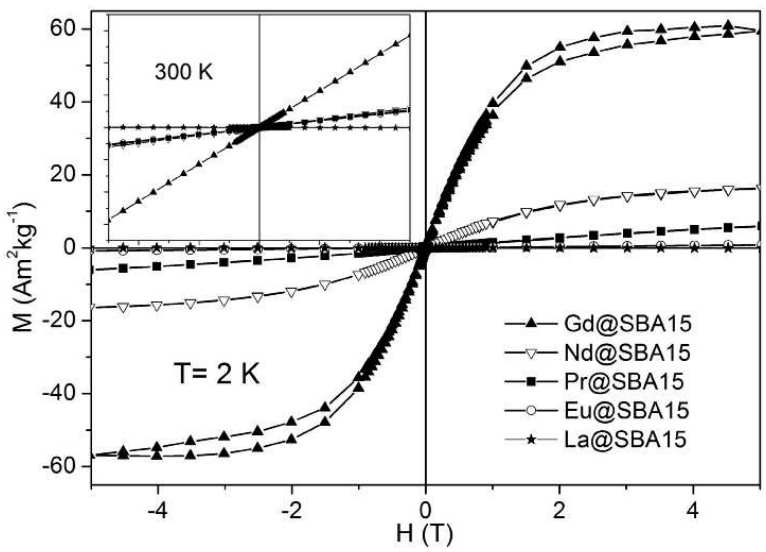

Fig. 3. $\quad M$ vs $H$ of studied samples at $2 \mathrm{~K}$ and $300 \mathrm{~K}$ (inset).

\section{Conclusions}

We have prepared lanthanide oxide based nanoparticles, growth of which was controlled by the rigid nanoporous silica template. The encapsulation of nanoparticles inside pores of SBA15 silica and their phase composition was confirmed by structural characterisation. Magnetic study showed the weak antiferromagnetic ordering at low temperature in $\mathrm{Ln}_{2} \mathrm{O}_{3} @$ SBA15 samples. Such long-range ordering at $2 \mathrm{~K}$ can be explained by the existence of superparamagnetic particles, which magnetic moments are blocked below critical temperature.

\section{Acknowledgments}

This work was supported by the VEGA No. 1/0583/11 project and the grants APVV-0222-10 and APVV-013211. The authors (A.Z and V.Z) would like to thank DESY/HASYLAB project under No. I-20110282 EC.

\section{References}

[1] S. Wang, Micr. Mesop. Mat. 117, 1 (2009).

[2] Y. Lin, Y. Hung, J. Su, R. Lee, C. Chang, M. Lin, C. Mou, J. Phys. Chem. B 108, 15608 (2004).

[3] M. Ballem, F. Soderlind, P. Nordblad, P.O. Kall, M. Oden, Micr. Mesop. Mat. 168, 221 (2013).

[4] D. Zhao, Q. Huo, J. Feng, B.F. Chmelka, G.D. Stucky, J. Am. Chem. Soc. 120, 6024 (1998).

[5] L.X. Chen, T. Liu, M.C. Thurnauer, R. Csencsits, T.J. Rajh, J. Phys. Chem. B 106, 8539 (2002). 